\title{
A Novel Measurement Approach for the Half-wave Voltage of Phase Modulator based on PM-MZI Photonic Link
}

\author{
Li Xianghua, Yang Chun*, Ye Quanyi, Chong Yuhua, and Zhou Zhenghua \\ School of Electronic Science and Engineering, Southeast University, Nanjing, Jiangsu Province, China
}

(Received March 3, 2014 : revised May 9, 2014 : accepted May 29, 2014)

\begin{abstract}
This paper presents a new method for measuring the half-wave voltage $V_{\pi}$ of an electro-optic phase modulator based on a phase-modulated photonic link with interferometric demodulation. By using this method, the $V_{\pi}$ can be obtained with the RF voltage amplitude input required to achieve 1-dB gain compression of link and the differential delay of a Mach-Zehnder interferometer. We measure the $V_{\pi}$ of a commercial phase modulator by using the presented method and the carrier/the first sideband intensity ratio method. Furthermore, we compare the two measurements with the typical value provided by the manufacturer. The experiment shows that this novel measurement method is feasible, straightforward, and accurate.
\end{abstract}

\begin{abstract}
Keywords : Half-wave voltage, Phase modulator, Microwave photonic link
OCIS codes : (250.4110) Modulators; (120.5060) Phase modulation; (060.2360) Fiber optics links and subsystems
\end{abstract}

\section{INTRODUCTION}

The use of photonic approaches to transmit and process microwave signals has been an interesting research area for several decades, due to its advantages of large bandwidth, low loss, and immunity to electromagnetic interference (EMI) [1]. Owing to the more linear conversion of input voltage to optical phase and requiring no bias controller, the $\mathrm{LiNbO}_{3}$ electro-optic phase modulator (PM) attracts a great deal of attention and has a host of applications [2, 3], such as microwave photonic links [4], optoelectronic oscillators [5, 6], optical comb generators, and all-optical microwave filters [7]. The half-wave voltage $\left(V_{\pi}\right)$ is one of the most significant parameters to characterize a PM, which is the voltage required to produce $\pi$ radians optical phase shift and represents the modulation efficiency of the PM. Therefore, the measurement of $V_{\pi}$ is important for PM manufacturers and end users.

Recently, several different methods for measuring the $V_{\pi}$ of a PM in the optical domain using an optical spectrum analyzer (OSA) have been proposed, such as the carrier nulling method [8], sideband nulling method, Carrier/the first sideband (FSB) intensity equalization method and Carrier/ FSB intensity ratio method [9]. Nevertheless, most of these methods require high driving power with peak-to-peak voltage significantly higher than $V_{\pi}$, which may change optoelectronic properties of PM and damage the device under test. And it is inconvenient to fine-tune the RF power manually at each frequency point to meet the critical measurement condition. Moreover, the resolution of commercial OSA is generally larger than $2 \mathrm{GHz}$, so that it is hard to measure the $V_{\pi}$ of PM in the lower frequency range by using these methods.

The method for measuring $V_{\pi}$ of $\mathrm{PM}$ in the electric domain based on the gain of phase-modulated link is also demonstrated [10]. Although this method eliminates the manual adjustment of RF power at each frequency point and does not require high driving power, lots of factors that have to be calibrated beforehand may degrade the measurement accuracy, such as the optical power of laser, optical insertion loss and responsivity of photodiode (PD). Even worse, the responsivity of PD is also frequencydependent and it is hard to calibrate as well.

In this paper, we propose a method for measuring the $V_{\pi}$ of a PM based on a phase-modulated Mach-Zehnder interferometric demodulation (PM-MZI) photonic link. In the measurement, the $V_{\pi}$ only depends on the RF voltage amplitude input required to achieve 1-dB gain compression

*Corresponding author: yangchun_seu@163.com 
of the link $\left(V_{R F, i n, I d B}\right)$ and the differential delay of Mach-Zehnder interferometer (MZI). And the $V_{R F, i n, I d B}$ can be automatically swept using a network analyzer (NA), eliminating the manual adjustment of RF power at every frequency point. The $V_{R F, i n, I d B}$ of the link can be reached at low driving power, which ensures the safe operation of the PM under test. Moreover, $V_{\pi}$ can be measured in the low frequency range. Additionally, this method does not depend on the frequency response of the PD.

\section{PRINCIPLE}

The Fig. 1 shows a PM-MZI link which comprises a laser, an external PM, an asymmetric MZI and a PD. Firstly, the signal is modulated on the phase of optical carrier by the PM. Then, the optical carrier is fed into the MZI which converts phase modulation into intensity modulation. And finally, the RF signal can be directly recovered by a PD. The photocurrent output of the PD is given as follows with the MZI set at quadrature [11]:

$$
I(t)=I_{d c}\{1+\sin [\phi(t)-\phi(t-\tau)]\}
$$

where $I_{d c}=a P_{0} \Re / 2, a$ is the total optical insertion loss of the link, $P_{0}$ is the optical power output of the laser, $\Re$ is the PD responsivity, $\tau$ is the differential delay of the MZI, and $\phi(t)=\pi V_{R F} \sin (\omega t) / V_{\pi}$ with $V_{R F} \sin (\omega t)$ as the driving signal.

The photocurrent given in the Eq. (1) can be expanded using the Bessel function of the first kind [12] and it is given by

$$
\begin{aligned}
I(t)= & I_{d c}+2 I_{d c} \sum_{n=1}^{+\infty} J_{2 n-1}\left[\frac{2 \pi V_{R F}}{V_{\pi}} \sin (\omega \tau / 2)\right] \\
& \sin [(2 n-1) \omega t]
\end{aligned}
$$

where $J_{2 n-1}$ is the (2n-1)th order Bessel function of the first kind. According to the Eq. (2), the RF power output at the fundamental frequency $\left(P_{R F, \text { out }}\right)$ of the link can be derived with $n=1$. And it is given by

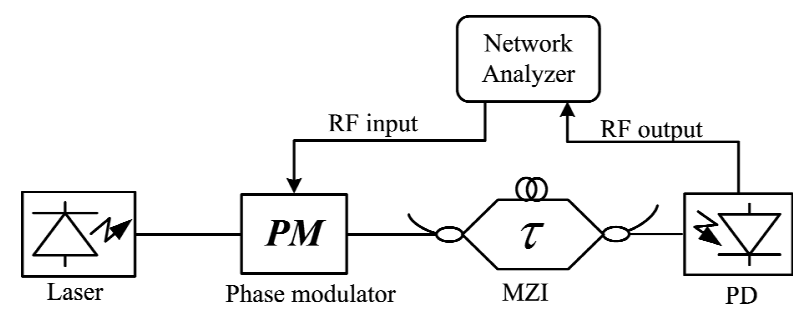

FIG. 1. The schematic diagram of half-wave voltage measurement.

$$
P_{R F, \text { out }}=2\left\{I_{d c} J_{1}\left[2 \frac{\pi V_{R F}}{V_{\pi}} \sin (\omega \tau / 2)\right]\right\}^{2} Z_{L}
$$

where $Z_{L}$ is the output impedance. Owing to the nonlinearity of the first order Bessel function, the $P_{R F, \text { out }}$ will be compressed when the RF power input is large enough. Generally, the small signal approximation of the RF power output at the fundamental frequency $\left(P_{R F, \text { out ss }}\right)$ can be obtained using the linear approximation of the 1st order Bessel functions $J_{l}(x)=x / 2$. And it is given as follows:

$$
P_{R F, o u t, s s}=2\left[I_{d c} \frac{\pi V_{R F}}{V_{\pi}} \sin (\omega \tau / 2)\right]^{2} Z_{L}
$$

With $P_{R F, i n}=V_{R F}^{2} /\left(2 Z_{i n}\right)$ and Eq. (4), the small-signal RF gain of the PM-MZI link is

$$
G=4\left[I_{d c} \frac{\pi}{V_{\pi}} \sin (\omega \tau / 2)\right]^{2} Z_{i n} Z_{L}
$$

As can be seen from Eq. (5), the PM-MZI link exhibits a frequency-dependent gain dictated by the differential delay of MZI. And the free spectral range (FSR) of the gain is equal to the reciprocal of the differential delay of MZI $(F S R=1 / \tau)$.

With the quotient (4) over (3) set to $10^{0.1}(1 \mathrm{~dB})$, Eq. (6) can be obtained. In this case, the $V_{R F}$ equals the RF voltage amplitude input required to achieve 1-dB gain compression $\left(V_{R F, i n, I d B}\right)$ of the link.

$$
\frac{\pi V_{R F} \sin (\omega \tau / 2) / V_{\pi}}{J_{1}\left[2 \pi V_{R F} \sin (\omega \tau / 2) / V_{\pi}\right]}=10^{0.05}
$$

In order to find the relational expression of $V_{\pi}$, we expand the first order Bessel function of the first kind to the ninth-order polynomial based on the following formula [12]

$$
J_{n}(x)=\sum_{l=0}^{\infty} \frac{(-1)^{l}}{2^{2 l+n} l !(n+l) !} x^{2 l+n}
$$

Consequently, an eighth-degree polynomial equation with the unknown of $V_{\pi}$ is obtained. The relational expression of $V_{\pi}$ can be found by numerically solving this eighth-degree polynomial equation and is given by

$$
V_{\pi}=2.104 \pi \sin (\omega \tau / 2) V_{R F, i n, l d B}
$$

As can be seen from Eq. (8), the $V_{\pi}$ of the PM can be calculated directly with the $V_{R F, i n, I d B}$ and the differential 
delay of the MZI. Importantly, the measurement of $V_{\pi}$ is independent of the responsivity of $\mathrm{PD}$, the optical power as well as the optical insertion loss.

The RF voltage amplitude input required to drive the link into $1-\mathrm{dB}$ compression is related to $V_{\pi}$ by

$$
V_{R F, i n, 1 d B} \cong \frac{0.15}{\sin (\omega \tau / 2)} V_{\pi}
$$

Obviously, the smallest peak-to-peak voltage swings 30\% of $V_{\pi}$ when $|\sin (\omega \tau / 2)|=1$. The required peak-to-peak voltage input based on this method is much smaller than for the previous methods, such as carrier nulling method $\left(V_{R F, i n, p p}\right.$ $\left.\cong 1.53 V_{\pi}\right)$ [8], sideband nulling method $\left(V_{R F, \text { in }, p p} \cong 2.439 V_{\pi}\right)$, and Carrier/FSB intensity equalization method $\left(V_{R F, i n, p p} \cong\right.$ $0.914 V_{\pi}$ ) [9]. Therefore, the method presented here with low RF drive voltage ensures the safe operation of the PM.

\section{EXPERIMENT}

The experimental setup of $V_{\pi}$ measurement is shown in Fig. 1, which is constructed with an Emcore 1772 DFB laser, a Covega LN53 PM, a DSC40S-HLPD PD and an asymmetric MZI. We sweep and normalize the gain of the PM-MZI link by using a network analyzer (Agilent PNA-X N5242A). The FSR of the gain is measured and it is equal to $3.37 \mathrm{GHz}$, as shown in Fig. 2 .

The Eq. (8) shows that the $V_{\pi}$ can be calculated directly from the $V_{R F, i n, l d B}$ and the differential delay of MZI. According to $F S R=1 / \tau$, the differential delay of MZI can be calculated and it is equal to 297 ps. Therefore, the $V_{R F, i n, I d B}$ is required to be measured. The N5242A network analyzer could automatically track the $1-\mathrm{dB}$ gain compression point and the RF power input required to achieve 1-dB gain compression $\left(P_{R F, i n, I d B}\right)$. With the measured $P_{R F, i n, I d B}$, the $V_{R F, \text { in, IdB }}$ can be calculated by using $V_{R F, \text { in, IdB }}=\sqrt{P_{R F, \text { in, ldB }} /\left(2 Z_{\text {in }}\right)}$.

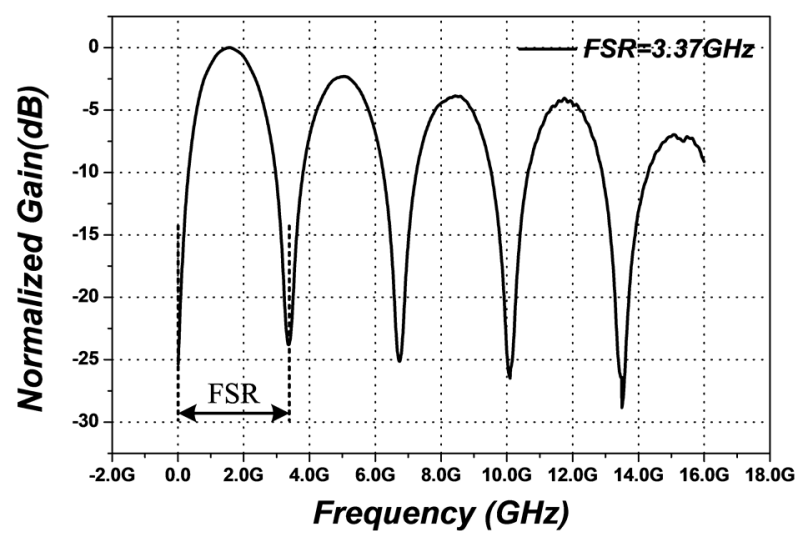

FIG. 2. The normalized gain of the PM-MZI link with FSR = $3.37 \mathrm{GHz}$.
We measure the $P_{R F, \text { in }, I d B}$ below $16 \mathrm{GHz}$ with different optical power outputs of laser $\left(P_{0}=60.1 \mathrm{~mW}, 40.2 \mathrm{~mW}\right.$ and $20.8 \mathrm{~mW}$ ). The error bar chart of $P_{R F, \text { in, IdB }}$ is shown in Fig. 3.

As shown in Fig. 3, the $P_{R F, i n, I d B}$ of the PM-MZI link is frequency-dependent and does not rely on the optical power output of the laser, which agrees well with Eq. (9). The frequency-dependent $P_{R F, i n, l d B}$ is dictated by the differential delay of MZI. Usually, with small MZI differential delay, the $P_{R F, i n, I d B}$ varies gently with frequency due to the large FSR. But $P_{R F, i n, I d B}$ can not be measured at low frequency because it is so large that it exceeds the maximum output power of the network analyzer. Whereas, with large MZI differential delay, the $P_{R F, i n, 1 d B}$ swings frequently with frequency because of small FSR. And more frequency points meet $|\sin (\omega \tau / 2)|=1$. Therefore, the MZI with large differential delay is recommended for our $V_{\pi}$ measurement method.

According to the measured $P_{R F, i n, I d B}$ in Fig. 3, the $V_{R F, i n, I d B}$ can be obtained. Then, the $V_{\pi}$ of PM can be calculated with $V_{R F, i n, I d B}$ and the differential delay of MZI $\tau$ based on Eq.

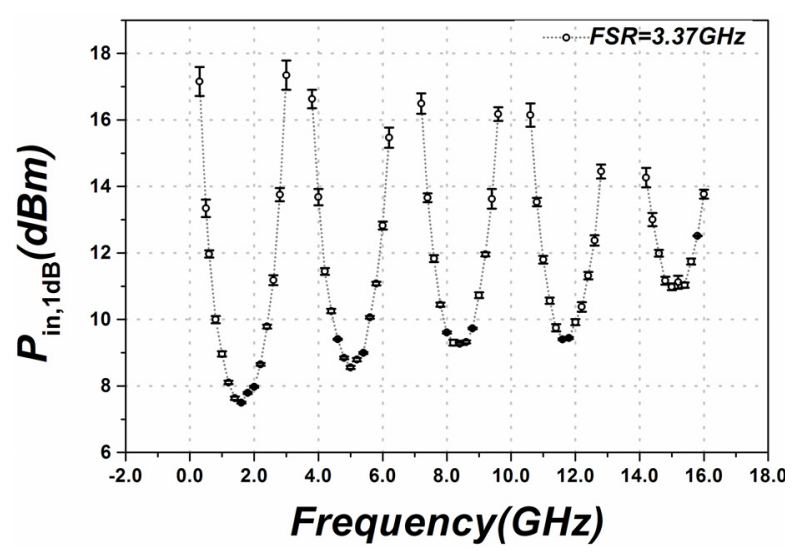

FIG. 3. The error bar chart of $P_{R F, i n, l d B}$, which is measured below $16 \mathrm{GHz}$ with different optical power outputs of the laser $\left(P_{0}=60.1 \mathrm{~mW}, 40.2 \mathrm{~mW}\right.$ and $\left.20.8 \mathrm{~mW}\right)$.

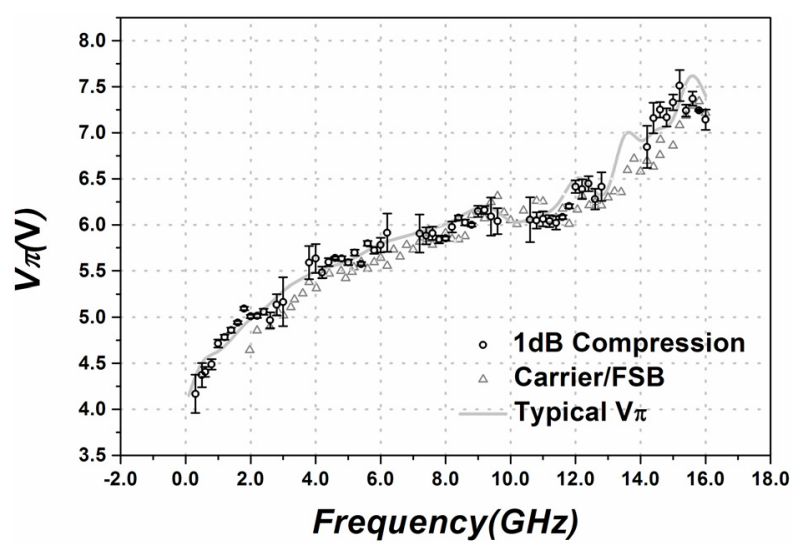

FIG. 4. The measured V $\pi$ of phase modulator and the typical $\mathrm{V} \pi$ provided by the manufacturer. 
(8). Furthermore, we also measure the $V_{\pi}$ of the PM using OSA (YOKAGAWA AQ6370) based on the carrier/FSB intensity ratio method [9]. The $V_{\pi}$ measurements based on the novel method (circles) and the carrier/FSB intensity ratio method (triangles) are shown in Fig. 4. As can be seen, the two $V_{\pi}$ measurements and the typical value of $V_{\pi}$ provided by the manufacturer increase with frequency and vary between $4 \mathrm{~V}$ and $8 \mathrm{~V}$ below $16 \mathrm{GHz}$. Overall, the two $V_{\pi}$ measurements agree well with the manufacturer's typical value. The measurement error of carrier/FSB intensity ratio method is less than $0.4 \mathrm{~V}$. However, the measurement error of the proposed method is not more than $0.2 \mathrm{~V}$ at the frequency that $|\sin (\omega \tau / 2)|$ is close to 1 , and less than $0.4 \mathrm{~V}$ at the frequency that $|\sin (\omega \tau / 2)|$ is away from 1 . Therefore, compared with the carrier/FSB intensity ratio method, this method exhibits high accuracy, especially when the measurement is implemented with a large MZI differential delay because more frequency points meet $|\sin (\omega \tau / 2)|=1$.

\section{CONCLUSION}

In this paper, we report a novel and simple method for measuring the $V_{\pi}$ of a PM based on PM-MZI photonic link. The theory shows that the $V_{\pi}$ of a PM can be calculated directly with $V_{R F, i n, I d B}$ and the differential delay of MZI. In the experiment, we measure the $V_{\pi}$ of a PM using the proposed method and the carrier/FSB intensity ratio method, respectively. And the two $V_{\pi}$ measurements agree well with the typical value provided by the manufacturer.

Compared with the previously reported methods, this novel method avoids the process of manual adjustment of $\mathrm{RF}$ power and eliminates the dependence on responsivity of PD, optical power and optical insertion loss. Moreover, Based on this method, the $V_{\pi}$ of a PM can be measured in the low frequency range and with low lever RF driving amplitude, smaller than $0.5 V_{\pi}$, which ensures the safe operation of the device under test. Therefore, it is an attractively alternative method to measure the $V_{\pi}$ of PM.

\section{REFERENCES}

1. R. C. Williamson and R. D. Esman, "RF photonics," J. Lightwave Technol. 26, 1145-1153 (2008).

2. V. J. Urick, F. Bucholtz, J. D. McKinney, P. S. Devgan, A. L. Campillo, J. L. Dexter, and K. J. Williams, "Long-haul analog photonics," J. Lightwave Technol. 29, 1182-1205 (2011).

3. V. J. Urick, F. Bucholtz, P. S. Devgan, J. D. McKinney, and K. J. Williams, "Phase modulation with interferometric detection as an alternative to intensity modulation with direct detection for analog-photonic links," IEEE Trans. Microwave Theory Tech. 55, 1978-1985 (2007).

4. J. D. McKinney and K. J. Williams, "A linearized phasemodulated analog optical link," in Proc. Conf. Quantum Electron. Laser Sci. Conf. Lasers Electro-Opt. (San Jose, CA, United States, May 2008), pp. 235-236.

5. T. Sakamoto, T. Kawanishi, and M. Izutsu, "Optoelectronic oscillator using a $\mathrm{LiNbO}_{3}$ phase modulator for self-oscillating frequency comb generation," Opt. Lett. 31, 811-813 (2006).

6. Y. Chong, C. Yang, X. Li, and Q. Ye, "A frequency-doubling optoelectronic oscillator using a three-arm dual-output MachZehnder modulator," J. Opt. Soc. Korea 17, $491-493$ (2013).

7. Z. Fei and Y. Jianping, "Investigation of phase-modulatorbased all-optical bandpass microwave filter," J. Lightwave Technol. 23, 1721-1728 (2005).

8. R. Tench, J. M. P. Delavaux, L. Tzeng, R. W. Smith, L. L. Buhl, and R. Alferness, "Performance evaluation of waveguide phase modulators for coherent systems at 1.3 and 1.5 um," J. Lightwave Technol. 5, 492-501 (1987).

9. S. Yongqiang, Y. Lianshan, and A. E. Willner, "High-speed electrooptic modulator characterization using optical spectrum analysis," J. Lightwave Technol. 21, 2358-2367 (2003).

10. Q. Ye, C. Yang, Y. Chong, and X. Li, "An alternative phase modulator half-wave voltage measurement based on photonic link," Chin. Opt. Lett. 11, 71202 (2013).

11. J. D. McKinney, K. Colladay, and K. J. Williams, "Linearization of phase-modulated analog optical links employing interferometric demodulation," J. Lightwave Technol. 27, 1212-1220 (2009).

12. F. W. J. Oliver, "Bessel functions of integer order," in Handbook of Mathematical Functions (1972), pp. 355-434. 